\title{
Oncogenic function of Plac1 on the proliferation and metastasis in hepatocellular carcinoma cells
}

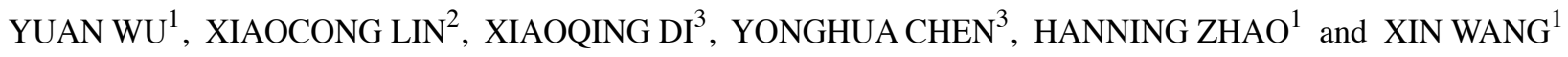 \\ Departments of ${ }^{1}$ Microbiology and Immunology, and ${ }^{2}$ Biochemistry and Molecular Biology, \\ Guangdong Medical University, Zhanjiang, Guangdong 524023; ${ }^{3}$ Department of Pathology, \\ Affiliated Hospital of Guangdong Medical University, Zhanjiang, Guangdong 524001, P.R. China
}

Received June 8, 2016; Accepted October 31, 2016

DOI: $10.3892 /$ or.2016.5272

\begin{abstract}
Placenta-specific protein 1 (Plac1), which is selectively expressed in the placental syncytiotrophoblast in adult normal tissues, plays an essential role in normal placental and embryonic development. Accumulating evidence suggests that enhanced Placl expression is closely associated with the progression of human cancer. Whether Plac1 contributes to the pathophysiology of hepatocellular carcinoma (HCC) remains unclear. In the present study, our data revealed that the expression of Plac1 in human HCC tissues was upregulated, which significantly correlated with metastasis of HCC. Knockdown of Plac1 by small interfering RNA (siRNA) in Bel-7402 and HepG2 cells resulted in decreasing tumor cell proliferation and increasing apoptosis, which implied the oncogenic potential of Plac1. Moreover, silencing of Plac1 induced G1 cell cycle arrest through suppression of cyclin D1 and CDK4 expression. Furthermore, depletion of Plac1 repressed epithelial-mesenchymal transition (EMT), with decreased cell migration and invasion, supporting upregulated E-cadherin expression and downregulated vimentin, twist and snail expression that characterize EMT. Further study suggested that decreased Placl expression attenuated the phosphorylation of Akt. These findings have uncovered that Placl plays a pivotal role in the progression of $\mathrm{HCC}$, and may serve as a novel therapeutic target for $\mathrm{HCC}$.
\end{abstract}

\section{Introduction}

Hepatocellular carcinoma (HCC) is the most common primary malignancy of the liver and the second leading cause of cancerrelated death worldwide. Globally, its incidence remains high,

Correspondence to: Dr Yuan Wu, Department of Microbiology and Immunology, Guangdong Medical University, No. 2 Wenming East Road, Xiashan, Zhanjiang, Guangdong 524023, P.R. China E-mail:wuyuan@gdmu.edu.cn

Key words: placenta-specific protein 1, hepatocellular carcinoma cell, epithelial-mesenchymal transition, metastasis, proliferation with approximately 750,000 new cases reported per year. Although significant improvement in diagnosis and treatment of HCC has been achieved, the prognosis for HCC patients who were surgically resectable remains poor. As estimated, the recurrence rate is approximately $50 \%$ in 2 years and $75 \%$ in 5 years after resection, respectively $(1,2)$, and most patients present with unresectable and advanced tumors. Therefore, it is vital to understand the underlying mechanism of tumor growth and metastasis and develop efficacious therapeutics for HCC.

Plac1, a known placenta-specific gene, is located on the X-chromosome (Xq26.3) (3). Plac1 gene encodes a 26-kDa protein, which is primarily expressed in cells of the trophoblastic lineage, and plays an important role in human placental development (4-6). Although the expression is mostly restricted to placenta in normal tissues, Plac1 is frequently activated and highly expressed in wide variety of human tumor cell lines and tissues, including breast, lung, colon, prostatic, cervical, gastric and hepatocellular cancer (7-10). Emerging evidence indicates that Plac1 is involved in the pathogenesis of cancer. Koslowski and coworkers (10) recently disclosed that knockdown of Plac1 suppressed breast cancer cell migration and invasion, and resulted in an interception of breast cancer cell proliferation by inducing a distinct G1 cell cycle arrest. Further, silencing of Plac1 decreased cyclin D1 expression and phosphorylation of AKT, which influenced cancer development. Since Placl has effects on the development of placenta and cancer, some scholars have given Plac1 designation cancer-placenta antigen 1 (CP1), which is regarded as a promising candidate for targeted immunotherapy of cancer $(7,8)$. Some surveys revealed that Placl elicited spontaneous antibody responses in HCC (8), and triggered anticancer immune responses by inducing the generation of Plac1-reactive antibodies and $\mathrm{T}$ cells in colorectal cancer patients (7). Nevertheless, the regulatory mechanism of Plac1 in the development of HCC is still not well elucidated.

In the present study, we discovered that Plac1 expression was significantly increased in HCC tissues, and associated with metastasis in HCC patients. Moreover, Placl was involved in the regulation of proliferation, cell cycle, apoptosis and EMT, which were correlated with AKT signaling pathway in HCC. Collectively, our findings indicate that Placl may be an effective therapeutic target for HCC. 


\section{Materials and methods}

Patients and tissues. Forty-six cases of paraffin-embedded HCC samples and non-cancerous liver tissues were obtained from the Department of Pathology of Affiliated Hospital of Guangdong Medical University. All the specimens were validated by pathological diagnosis complying with the World Health Organization standards. This study was conducted with the approval of the Medical Ethics Committee of Affiliated Hospital of Guangdong Medical University.

Cell lines. HepG2, Bel-7402, SMMC-7721, Huf7 and L02 cells were obtained from the Cell Blank of Shanghai Biology Institute, Chinese Academy of Sciences (Shanghai, China). HLE cell line was a gift from Professor Yanhui Yin (Peking University Health Science Center, Beijing, China). These cells were cultured in Dulbecco's modified Eagle's medium (DMEM; HyClone Laboratories, Thermo Fisher Scientific, Waltham, MA, USA) and supplemented with $10 \%$ fetal bovine serum (FBS; HyClone Laboratories, Thermo Fisher Scientific) in a humidified incubator at $37^{\circ} \mathrm{C}$ with $5 \% \mathrm{CO}_{2}$.

Cell transfection. The HepG2 and Bel-7402 cells were assigned to Plac1-siRNA group, blank group, and negative control group (NC siRNA). Cells in Placl-siRNA (Sigma-Aldrich, St. Louis, MO, USA) group were transfected with the following sequences: 5' to 3'-GUGUUCAGCUUGUCACAGU, antisense, 5' to 3 ' ACUGUGACAAGCUGAACAC. Cells in the negative control group were transfected with NC siRNA, which was a non-specific scramble siRNA. Cells in the blank group were exposed to normal medium. For transient transfection, HepG2 and Bel-7402 cells were transfected with siRNA using Lipofectamine 2000 (Invitrogen, Carlsbad, CA, USA) following the manufacturer's instruction. Four hours after the transfection, cell were transferred to fresh medium-containing $10 \%$ FBS and incubated for 24-36 h.

Immunohistochemistry. Tissue slides were dewaxed according to the standard protocol. Paraffin sections of HCC were deparaffinized, hydrated and washed in phosphate-buffered saline (PBS). After pretreatment in a microwave oven for $8 \mathrm{~min}$, the endogenous peroxidase was inhibited with $3 \% \mathrm{H}_{2} \mathrm{O}_{2}$ for $10 \mathrm{~min}$, and the sections were incubated with $10 \%$ normal goat serum for $30 \mathrm{~min}$. Subsequently, the sections were incubated with Plac1 primary antibody (Abcam, 1:100) at $4^{\circ} \mathrm{C}$ overnight. After washing, the sections were incubated for 30 min with secondary antibody (Dako, Carpinteria, CA, USA) at room temperature. Following this incubation, the sections were washed three times in PBS, and the visualization signal was developed using diaminobenzidine tetrahydrochloride (DAB), followed by counterstaining with hematoxylin.

Western blot analysis. Cells were washed three times with cold PBS, and lysed in RIPA-buffer $(0.5 \%$ sodium deoxycholate, $0.1 \%$ SDS, $1 \%$ NP-40 and PBS) with PMSF and protease inhibitor cocktail (Sigma-Aldrich). Equal amount of protein $(30 \mu \mathrm{g})$ was separated by sodium dodecyl sulfate-polyacrylamide gel electrophoresis (SDS-PAGE) and transferred onto polyvinylidene fluoride (PVDF; Millipore Corp., Bedford, MA, USA) membrane after quantification.
Following incubation in 5\% BSA, the membranes were incubated with primary antibodies: CDK4 $(1: 1,000)$, snaill $(1: 1,000)$, AKT $(1: 1,000)$, p-AKT $(1: 2,000)$, E-cadherin $(1: 1,000)$, p53 $(1 ; 1,000)$ (Cell Signaling Technology, Danvers, MA, USA), Twist $(1: 1,000)$, cyclin D1 $(1: 1,000)$ (GeneTex, Inc., Irvine, CA, USA), Plac1 (1:1,000), vimentin $(1: 1,000)$, Bax (1:1,000), Bcl-2 (1:500) (Abcam, Cambridge, UK), and GAPDH $(1: 10,000)$ (KangChen Bio-tech Inc., Shanghai, China) overnight at $4^{\circ} \mathrm{C}$. Subsequently the membranes were incubated with a horseradish-peroxidase-conjugated secondary antibody (SouthernBiotech, Birmingham, AL, USA) at a dilution of 1:20,000 at room temperature for $1 \mathrm{~h}$ and visualized by chemiluminescence. GAPDH was used as an internal control for normalization.

Cell proliferation assay. Cell proliferation was performed using the Cell Counting kit-8 (CCK-8) assay (Beyotime Institute of Biotechnology, Shanghai, China) according to the manufacturer's instructions. After transfected with indicated siRNA, cells from Placl-siRNA group, blank group, and NC siRNA group were collected and seeded in 96-well plates ( $1 \times 10^{4}$ cells/well). Following further incubated for $0,24,48$ and $72 \mathrm{~h}$, respectively, the cells were incubated with CCK- 8 reagents $(10 \mathrm{ml} /$ well) for $4 \mathrm{~h}$. The optical density values (OD) were measured at $450 \mathrm{~nm}$. All experiments were performed at least three times.

Cell cycle analysis. For the cell cycle analysis, $1 \times 10^{6}$ cells from Placl-siRNA group, blank group, and NC siRNA group were collected after $48 \mathrm{~h}$, washed twice in cold PBS, and fixed with cold $70 \%$ ethanol at $4^{\circ} \mathrm{C}$ overnight. Then, the cells were washed in cold PBS, and resuspended in $500 \mu 1$ PBS with propidium iodide (PI), Triton X-100, and RNase A at $4^{\circ} \mathrm{C}$ for $30 \mathrm{~min}$. The samples were analyzed by a FACSCalibur flow cytometer (BD Biosciences, San Jose, CA, USA).

Cell apoptosis assay. Cell apoptosis was measured by FACS analysis using the Annexin V-FITC/PI apoptosis detection kit (Nanjing KeyGen Biotech, Co., Ltd., Nanjing, China). Cells $5 \times 10^{5}$ from Plac1-siRNA group, blank group, and NC siRNA group were collected and washed twice in cold PBS. Subsequently, the cells were resuspended in $500 \mu 1$ binding buffer, and incubated with $1.25 \mu \mathrm{l}$ Annexin V-FITC and $10 \mu \mathrm{l}$ propidium iodide in the dark at room temperature for $15 \mathrm{~min}$. The stained cells were analyzed by a FACSCalibur flow cytometer (BD Biosciences).

Cell migration and invasion assays. Cell migration and invasion were carried out by the way of Transwell and Matrigel invasion (BD Biosciences), respectively. For cell migration assay, $1 \times 10^{5}$ cells were seeded on the upper of chambers in serum-free DMEM. The lower chambers were filled with DMEM, supplemented with $10 \%$ FBS as a chemoattractant. After incubation for $48 \mathrm{~h}$, non-migrated cells on the top of the well were removed with cotton swabs. The cells migrated to the underside of the membrane were fixed with $4 \%$ paraformaldehyde for $15 \mathrm{~min}$, stained with $0.1 \%$ crystal violet, and counted under a microscope. For the invasion assay, the procedures were the same as those described above, except that the upper chambers were coated with Matrigel. Each experiment 


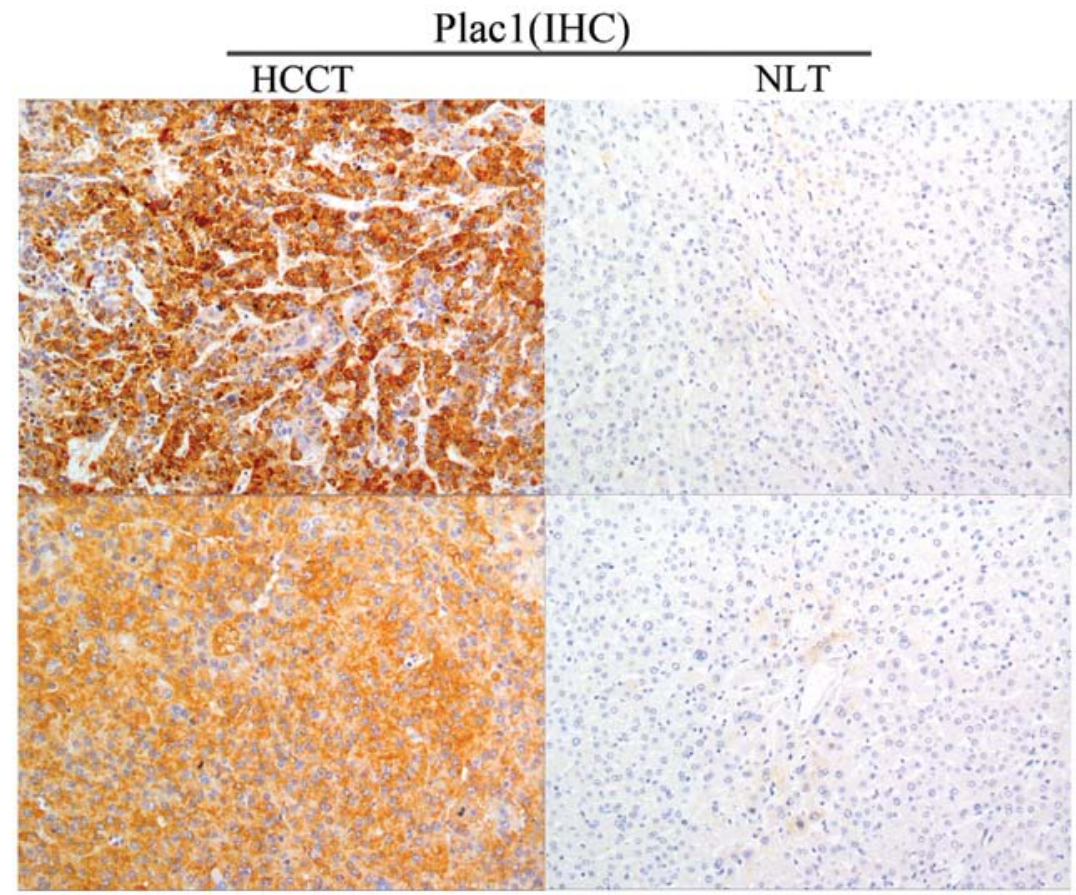

Figure 1. Plac1 is expressed in HCC tissues by immunohistochemical analysis. Positive expression of Plac1 in HCC tissue (HCCT) was examined. The expression of Plac1 in non-cancerous live tissue (NLT) was negative or weak.

was performed in triplicate and the mean value was calculated from three independent experiments.

Statistical analysis. SPSS 16.0 software was used for all the analysis. The correlation between the Plac1 and the individual clinicopathological factors was evaluated with the Chi-squared test. For other data, the two or multiple group comparisons were performed using the Student's t-test or ANOVA. Data were reported as mean $\pm \mathrm{SD}$. $\mathrm{P}<0.05$ was considered statistically significant.

\section{Results}

Placl is upregulated in HCC tissues and correlates with tumor metastasis. To clarify the underlying role of Plac1 in HCC progression, we first determined the protein expression level of Plac1 in 46 tumor specimens from HCC patients. Immunohistochemistry analysis revealed that Plac1 was highly expressed in $54.3 \%$ (25/46) of HCC tissues, and the protein was located diffusely in the cytoplasm and cell membrane. Noteworthy, non-cancerous liver tissues exhibited negative or weak Plac1 staining (Fig. 1). The correlation between the Placl expression level and the clinicopathological features was further evaluated, which is summarized in Table I. The results showed that Placl expression was not associated with the patient gender, age, HBsAg, serum AFP, tumor size or cirrhosis, but significantly with tumor metastasis $(\mathrm{P}=0.015)$. These data illustrate that Placl may be involved in the HCC pathogenesis and aggressiveness.

Placl is overexpressed in HCC cells and knocked down by siRNA. Next, we analyzed the Plac1 expression in five HCC cell lines, HLE, Bel-7402, SMMC-7721, HepG2 and Huh7 by western blot analysis. It was observed that Plac1 expression was
Table I. Relationship between the Plac1 expression and the clinicopathological features.

\begin{tabular}{|c|c|c|c|c|c|}
\hline Characteristics & $\begin{array}{l}\text { No. of } \\
\text { patients }\end{array}$ & $\begin{array}{l}\text { Plac1 } \\
(+)\end{array}$ & $\begin{array}{l}\text { Plac1 } \\
(-)\end{array}$ & $\chi^{2}$ & P-value \\
\hline \multicolumn{6}{|l|}{ Gender } \\
\hline Male & 36 & 19 & 17 & 0.002 & 0.963 \\
\hline Female & 10 & 6 & 4 & & \\
\hline \multicolumn{6}{|l|}{ Age (years) } \\
\hline$\leq 60$ & 30 & 14 & 16 & 2.051 & 0.152 \\
\hline$>60$ & 16 & 11 & 5 & & \\
\hline \multicolumn{6}{|l|}{$\mathrm{HBsAg}$} \\
\hline Positive & 36 & 19 & 17 & 0.096 & 0.757 \\
\hline Negative & 10 & 6 & 4 & & \\
\hline \multicolumn{6}{|l|}{$\mathrm{AFP}(\mu \mathrm{g} / \mathrm{l})$} \\
\hline$\leq 400$ & 24 & 12 & 12 & 0.382 & 0.536 \\
\hline$>400$ & 22 & 13 & 9 & & \\
\hline \multicolumn{6}{|l|}{ Cirrhosis } \\
\hline Absent & 33 & 17 & 16 & 0.378 & 0.539 \\
\hline Present & 13 & 8 & 5 & & \\
\hline \multicolumn{6}{|l|}{ Tumor size $(\mathrm{cm})$} \\
\hline$\leq 5$ & 23 & 10 & 13 & 2.190 & 0.139 \\
\hline$>5$ & 23 & 15 & 8 & & \\
\hline \multicolumn{6}{|l|}{ Differentiation } \\
\hline $\begin{array}{l}\text { Well and } \\
\text { moderate }\end{array}$ & 31 & 18 & 13 & 0.529 & 0.467 \\
\hline Poor & 15 & 7 & 8 & & \\
\hline \multicolumn{6}{|l|}{ Metastasis } \\
\hline Absent & 15 & 12 & 3 & 5.903 & 0.015 \\
\hline Present & 31 & 13 & 18 & & \\
\hline
\end{tabular}


A

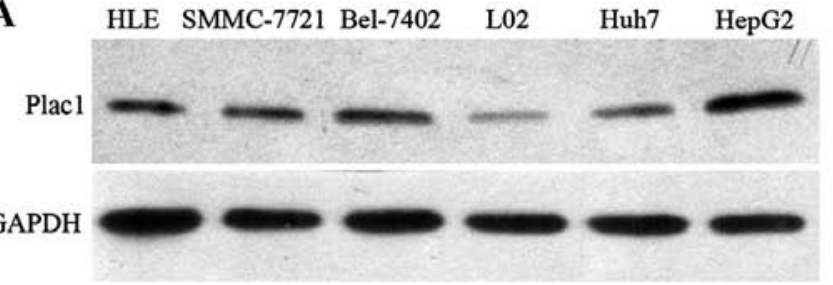

B

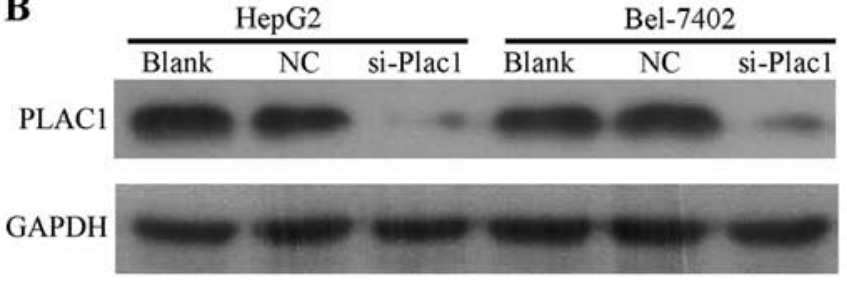

Figure 2. Plac1 is overexpressed in HCC cell lines. (A) The expression level of Plac1 in five HCC cell lines and normal liver cell line was analyzed by western blot analysis. (B) The silencing effects of Plac1-siRNA were evaluated by western blot analysis in HepG2 and Bel-7402 cells. Blank, cells exposed to normal medium; NC, cells transfected with scrambled siRNA; si-Plac1, cells transfected with Plac1-siRNA.

A

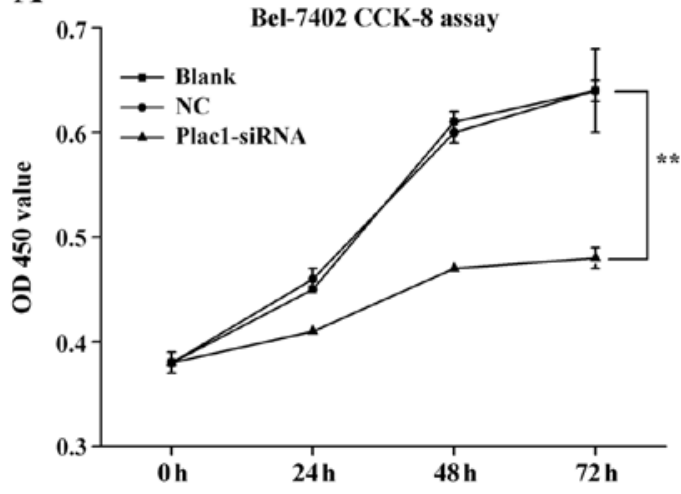

B

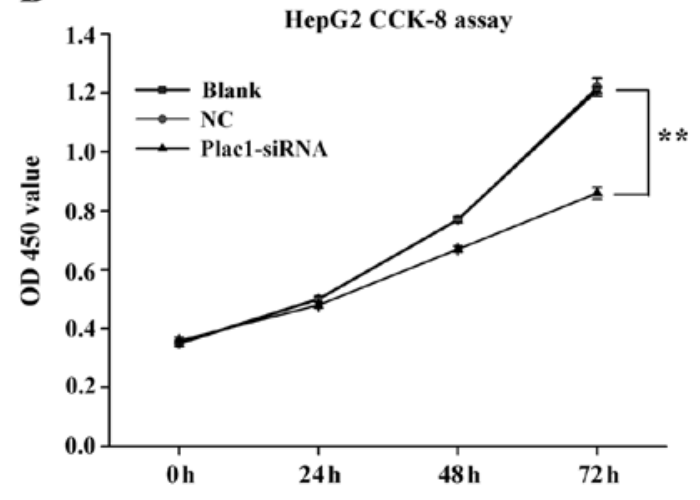

Figure 3. Knockdown of Plac1 inhibits cell proliferation in HCC cells. (A) The results of CCK-8 assay performed in Bel-7402 cells. (B) The results of CCK-8 assay performed in HepG2 cells. Blank, cells exposed to normal medium; NC, cells transfected with scrambled siRNA; Plac1-siRNA, cells transfected with Plac1-siRNA. Data are at least three independent experiments and are shown as mean $\pm \mathrm{SD}\left({ }^{* *} \mathrm{P}<0.01\right.$ as compared with $\left.\mathrm{NC}\right)$.

significantly upregulated in all HCC cell lines when compared to normal liver cell line (Fig. 2A). To further assess the impact of Plac1 on HCC, we employed Bel-7402 and HepG2 cells as model system, in which Placl expression is higher than other $\mathrm{HCC}$ cell lines. In these cells, we downregulated Placl expression by Plac1-specific siRNA, and a scrambled siRNA was used as a negative control. As expected, Plac1 protein expression was almost completely inhibited, as detected by western blotting (Fig. 2B).

Knockdown of Placl inhibits cell proliferation in HCC cells. We carried out cell proliferation assay to explore the effect of Plac1 on the growth of HCC cells. After Bel-7402 and HepG2 cells were transiently transfected with Plac1 specific siRNA (Plac1-siRNA) and a scrambled siRNA (siNC RNA, $\mathrm{NC}$, negative control) for $48 \mathrm{~h}$, they were evaluated by using CCK-8 assays. The cells treated with Plac1-siRNA exhibited a significant reduction in cell proliferation compared to control cells (Fig. 3). These data supported that Plac1 promotes the growth of HCC cells in hepatocarcinogenesis.

Downregulation of Placl causes cell cycle retardation and apoptosis of HCC cells. To verify if Plac1-siRNA-mediated suppression of cell growth is associated with cell cycle arrest or an induction of apoptosis, we performed cell cycle and apoptosis analysis using flow cytometry. We observed that the amount of apoptotic cells was more in cells transfected with Plac1-siRNA than those in control groups. The average percentage of apoptotic Bel-7402 cells in Plac1-siRNA, NC, and blank groups, was 45.96, 13.77 and $12.65 \%$, respectively, while apoptotic percentage was $30.84 \%$ in Plac1-siRNA HepG2 cells, $10.36 \%$ in NC HepG2 cells and $11.51 \%$ in blank HepG2 cells (Fig. 4A and B). To further investigate the molecular mechanism of these pro-apoptotic effects, we measured the expression of apoptosis-related proteins, including p53, Bcl-2 and Bax. In both Bel-7402 and HepG2 cells, silencing of Plac1 caused p53 protein level increased, while the pro-apoptotic protein Bax was downregulated. Notably, the anti-apoptotic protein Bcl-2 was increased in HepG2-siPlac1 cells, but reduced in Bel-7402-siPlac1 cells (Fig. 4G).

Next, we sought to determine cell cycle distribution after cells were treated with Plac1-siRNA or siNC RNA by flow cytometry. As shown in Fig. 4C-E, knockdown of Plac1 significantly enhanced the percentage of Bel-7402 and HepG2 cells in the G1 phase (52.64\% in Bel-7402 cells and $76.78 \%$ in HepG2 cells) in comparison to the NC (47.46\% in Bel-7402 cells and $69.13 \%$ in HepG2 cells) or blank group (46.72\% in Bel-7402 cells and $68.63 \%$ in HepG 2 cells), at the same time, accompanied by a decreased proportion of cells in $\mathrm{S}$ phase, which was decreased at least $10 \%$ in Bel-7402 cells and $36 \%$ in HepG2 cells, but G2 phase had no change. These results indicated that downregulation of Placl resulted in retardation of HCC cells in the G1 phase. To elucidate the mechanism of G1 accumulation induced by Plac1-siRNA, we used a western blot analysis to examine the expression levels of cyclin D1 and CDK4. Compared to the control groups, cells transfected with Plac1 siRNA exhibited inhibition of cyclin D1 and CDK4 expression (Fig. 4F). Therefore, these data demonstrated that 

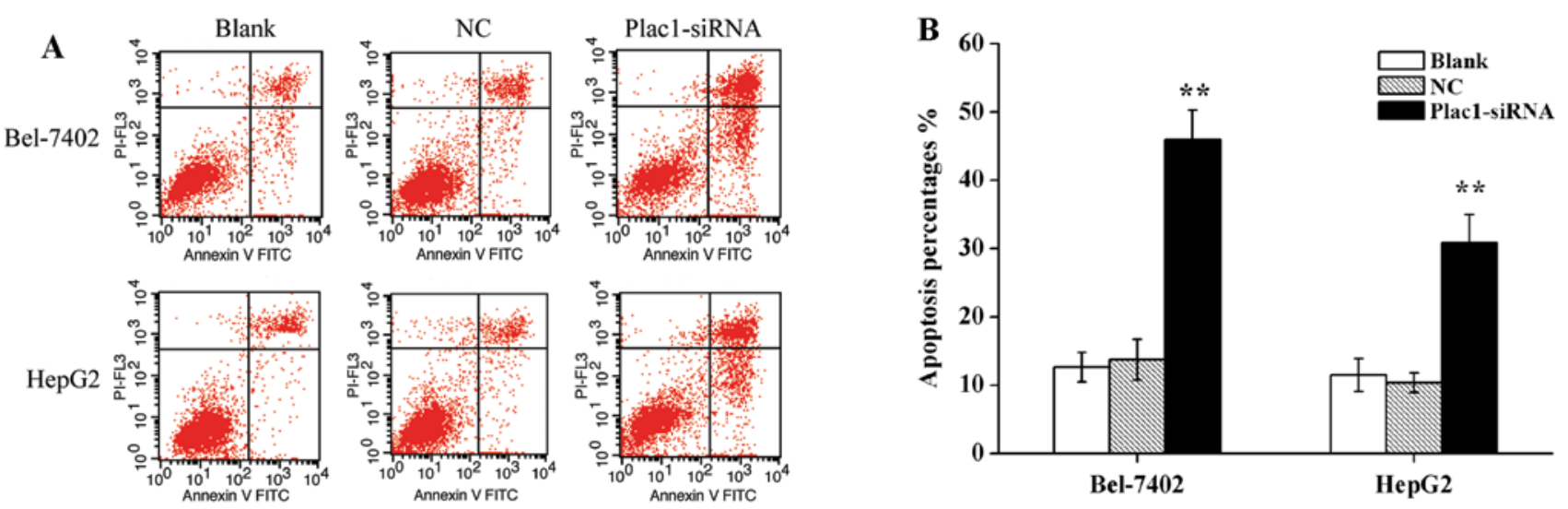

C

Bel-7402
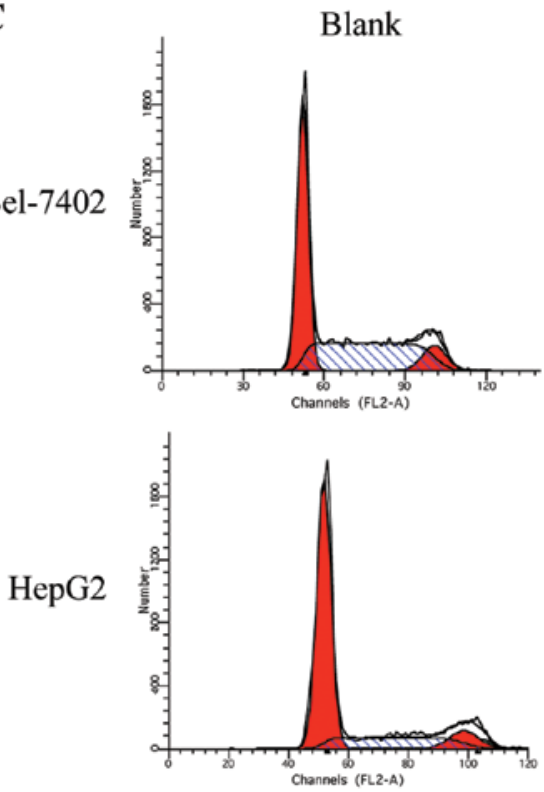

Bel-7402 cell cycle analysis

D

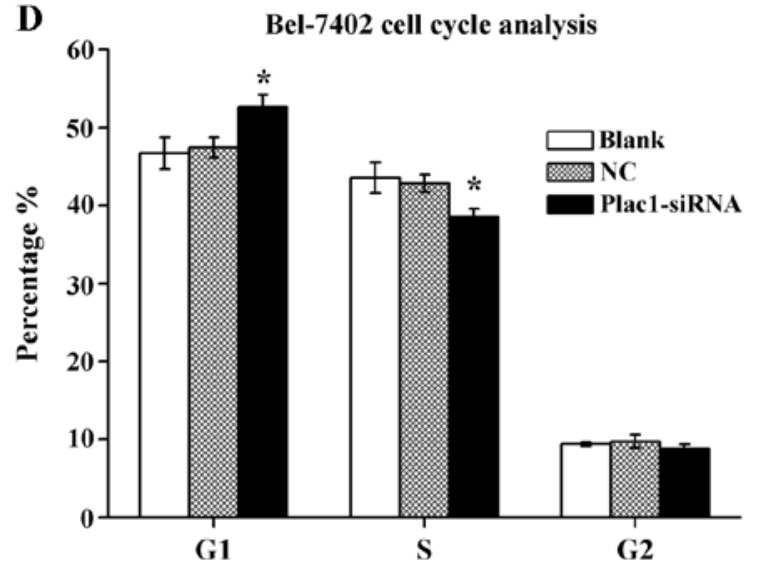

F

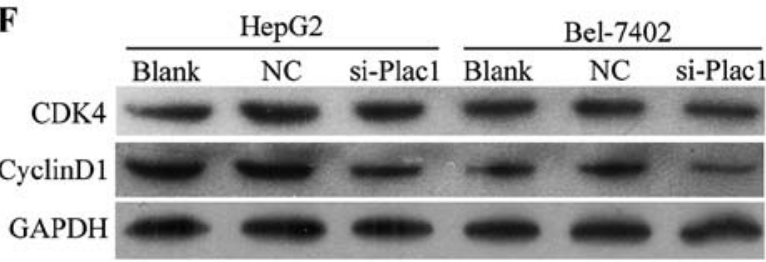

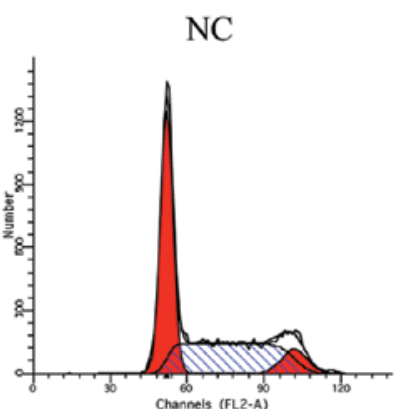
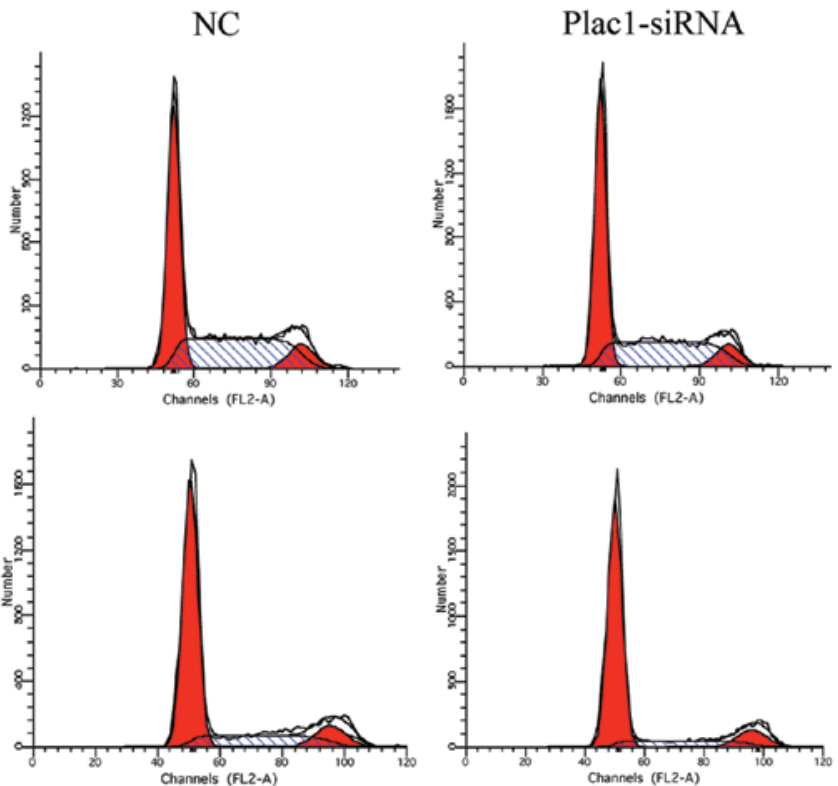

E HepG2 cell cycle analysis

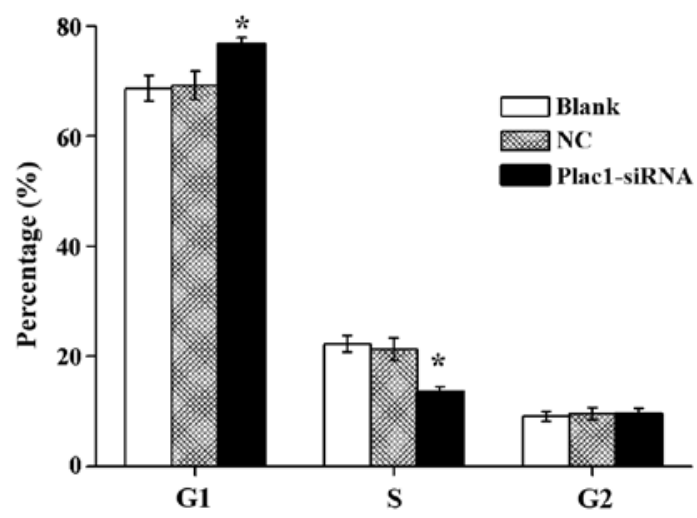

G

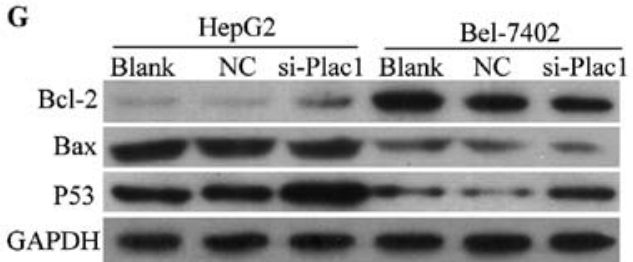

Figure 4. Silencing of Plac1 induced cell cycle arrest and cell apoptosis in Bel-7402 and HepG2 cells. (A and B) Depletion of Plac1 induced apoptosis in Bel-7402 and HepG2 cells ( ${ }^{* *} \mathrm{P}<0.01$ compared with NC). (C-E) Plac1-siRNA caused G1 retardation in Bel-7402 and HepG2 cells by FACS analysis ("P $<0.05$ compared with NC). (F and G) Western blot analysis showed apoptosis-associated protein and cycle-associated protein in Bel-7402 and HepG2 cells, respectively. Data are from at least three independent experiments and are shown as mean $\pm \mathrm{SD}$. ( ${ }^{* *} \mathrm{P}<0.01$ as compared with $\left.\mathrm{NC}\right)$. 
A

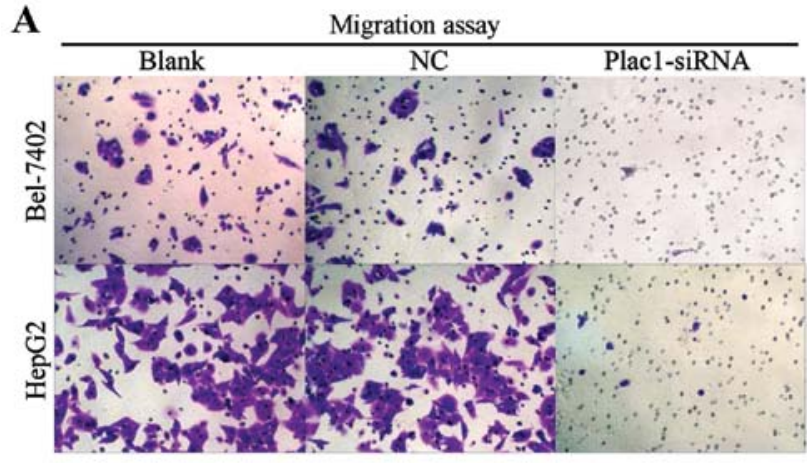

C

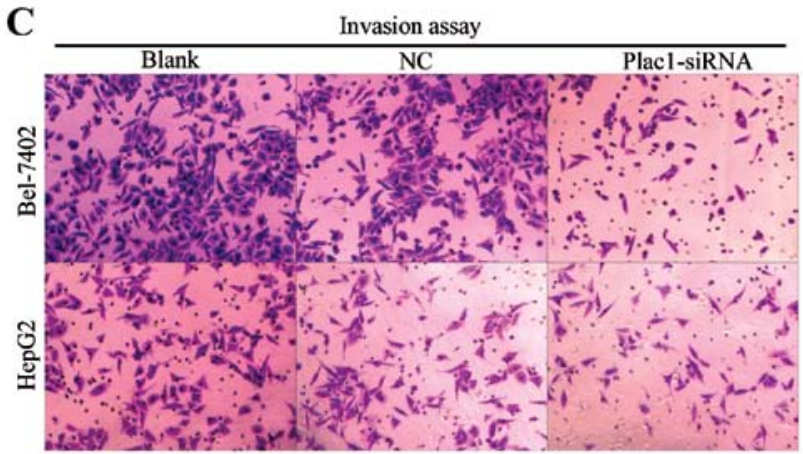

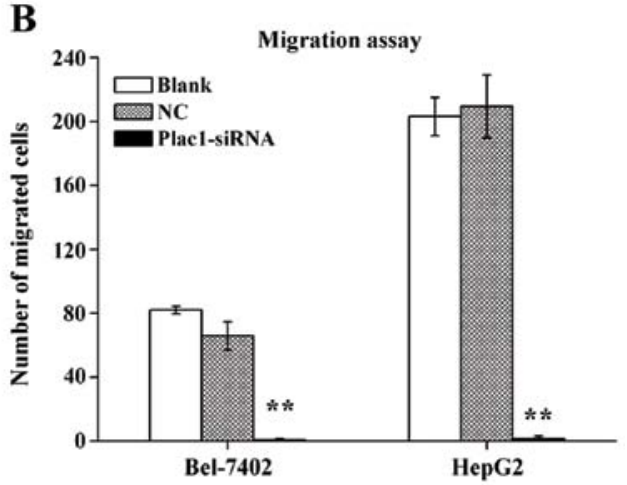

D

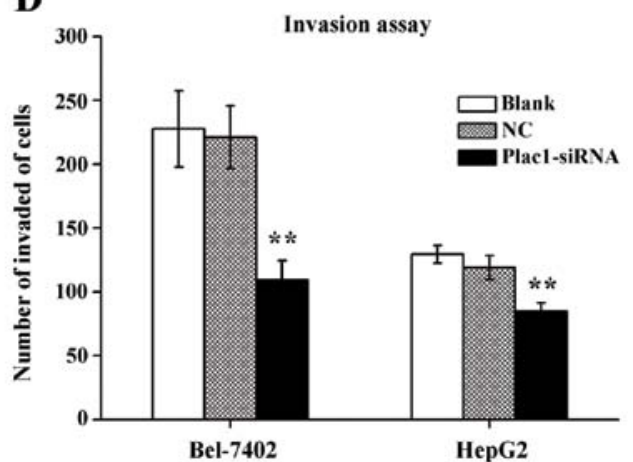

Figure 5. Downregulation of Plac1 reduced cell migration and invasion in Bel-7402 and HepG2 cells. (A and B) Migration assay in Transwell chambers (Bel-7402: $0.67 \pm 0.82$ vs. $82 \pm 2.28$ and $65.83 \pm 8.73$; HepG2: $1.5 \pm 1.64$ vs. 203.17 \pm 11.94 and 209.5 \pm 19.75 ). (C and D) Invasion assay in Matrigel-coated Transwell chambers (Bel-7402: 109.17 \pm 15.3 vs. 227.67 \pm 29.97 and 221 \pm 24.71 ; HepG2: $84.83 \pm 6.68$ vs. 129.33 \pm 6.92 and $119 \pm 9.36$ ). Data are from at least three independent experiments and are shown as mean $\pm \mathrm{SD}$. $\left({ }^{* *} \mathrm{P}<0.01\right.$ as compared with $\left.\mathrm{NC}\right)$.

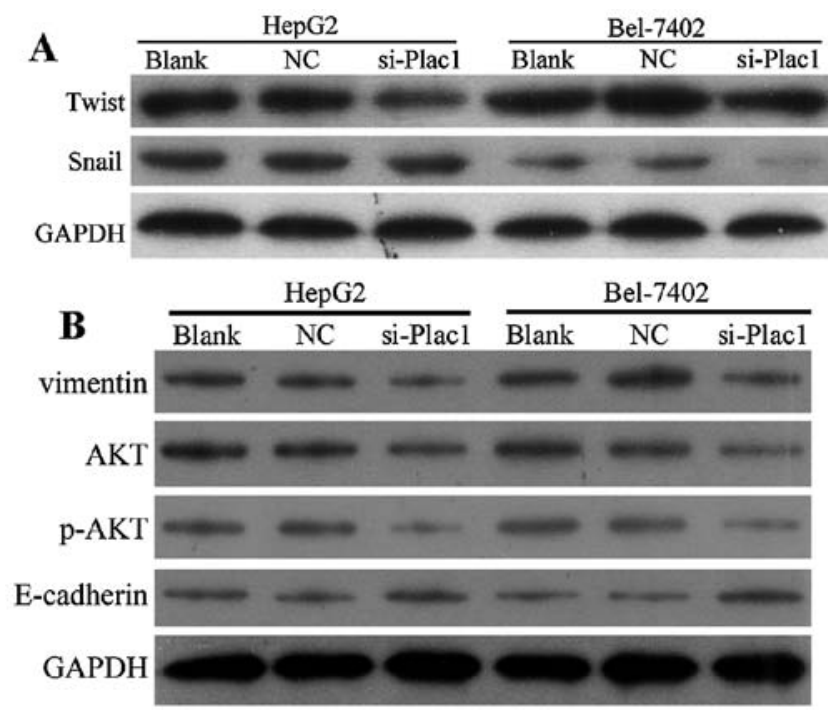

Figure 6. Depletion of Plac1 regulated EMT and p-AKT in HCC cells. (A) The expression of Snail and Twist was analyzed by western blotting. (B) Western blot analysis showed the expression level of E-cadherin, vimentin and p-AKT.

Placl enhanced cell proliferation through promoting cell cycle progression in HCC.

Decreased Placl expression represses cell migration and invasion in HCC cells. Because Plac1 overexpression was significantly associated with HCC metastasis, the effect of Plac1 in tumor cell migration and invasion was investigated.
Transwell migration assay revealed a distinctly impaired migratory ability in Plac1-siRNA transfected cells vs. control cells. The quantity of blank, NC and Plac1-siRNA cells, which migrated to the lower face of the Transwell membrane were $82 \pm 2.28,65.83 \pm 8.73$ and $0.67 \pm 0.82$ in Bel-7402 cells, respectively, and corresponding cell numbers were $203.17 \pm 11.94$, 209.5 \pm 19.75 and $1.5 \pm 1.64$ in HepG2 cells, respectively (Fig. 5A and B). Concordantly, knockdown of Placl also diminished cell invasion through Matrigel. As shown in Fig. 5C and D, Plac1-siRNA Bel-7402 cells demonstrated a decrease in the cell quantity $(109.17 \pm 15.3)$ vs. blank or NC cells $(227.67 \pm 29.97$ or $221 \pm 24.71$, respectively), while invaded cells were numbered $(84.83 \pm 6.68)$ in Plac1-siRNA HepG2 cells, (129.33 \pm 6.92 and

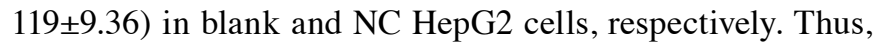
these findings confirmed that Placl promotes the motility and invasiveness of HCC cells.

Depletion of Placl suppresses EMT and leads to $p$-AKT reduction in HCC cells. As epithelial to mesenchymal transition (EMT) is one of the key events in tumor invasion and metastasis, we next conducted studies to investigate if EMT is responsible for Plac1-mediated change in cell motility. Compared with control cells, the epithelial marker E-cadherin expression had an increase in the cells treated with Plac1-siRNA, accompanied by a reduction of mesenchymal marker vimentin (Fig. 6B). Since Snail and Twist are transcription factors that have been demonstrated as key EMT regulators by suppressing the epithelial genes and inducing the mesenchymal genes, we further examined 
whether increased E-cadherin expression is the result of decreased Snail and Twist levels in the HCC cells treated with Plac1 siRNA. It is suggested that downregulation of Plac1 repressed Snail and Twist expression (Fig. 6A). Overall, our data are consistent with the hypothesis that depletion of Placl elevated E-cadherin protein level via inhibiting Snail and Twist expression. Considering the important regulator of AKT activation in promoting cancer cell proliferation, motility and invasiveness, we sought to evaluate the effect of Plac1 on AKT signaling in HCC. In Bel-7402 and HepG2 cells, decreased Placl expression markedly reduced phosphorylation of AKT (Fig. 6B). Thus, we interpret these data to support that Plac1 exerts carcinogenic effect possibly through the activation of AKT pathway.

\section{Discussion}

The incidence of HCC has been increased in the western and Asian countries. Although advances have been made in treatment of HCC with surgery, chemotherapy and radiotherapy, the mortality rate of HCC is still high. Good diagnostic markers, drug targets and therapeutic strategies are in urgent need for a successful treatment of HCC.

Plac1 is frequently expressed in the cells of the trophoblast lineage and in different types of malignant tumors (7-10). Additionally, overexpression of Plac1 is correlated with survival of gastric adenocarcinoma (11). Ghods et al (12) found that Plac1 presented differential expression in prostate cancer, and positivity was associated with the Gleason score, and negativity correlated with prostate specific antigen (PSA) expression, thus highlighting the potential usefulness of Plac1 for developing targeted prostate cancer therapies, particularly for patients with advanced disease. A recent study showed that Plac1 expression was absent in normal tissues except for placenta, and expressed in partial HCC samples, including mRNA and protein levels (8). Accordingly, we demonstrated that increased expression of Plac1 was observed in $54.3 \%$ of HCC samples and in several types of HCC cell lines, whereas non-cancerous liver tissues and normal liver $\mathrm{LO}_{2}$ cells barely expressed Placl in this study. Furthermore, HCC patients who had Placl expression had higher incidence of metastasis than those without Placl expression, which confirmed the effect of Placl on the migration and invasion of tumor cells. Taken together, these findings indicated that Placl may be an oncogene promoting HCC progression.

To address the pathobiological role of Plac1 in the HCC progess, we introduced Placl specific siRNA to silence Plac1 in Bel-7402 and HepG 2 cells, and assessed the impact of Plac1 on cell proliferation, cell cycle, apoptosis and invasiveness. The present study revealed that cell proliferation resulting from knockdown of Plac1 was suppressed. Moreover, downregulation of Plac1 in HCC cells resulted in a significant cell retardation in the $\mathrm{G} 1$ phase and increased apoptosis, which implicated a role of Plac1 in the resistance to apoptosis and enhancing HCC cell proliferation. These results provide further evidence to confirm Placl as a candidate oncogene in HCC. Notably, a study by Koslowski et al (10) showed that Plac1 had no an effect on apoptosis in breast cancer cell line MCF-7, the cell line variability may explain the differences between their finding and ours.
To elucidate how Plac1 affects the proliferation and cell cycle of HCC cells, we explored the levels of cell cyclerelated protein. Cyclin D1 known as cyclin, and CDK4 called the cyclin-dependent kinase, mediate cell proliferation and differentiation through regulating the G1/S transition of the cell cycle (13-15). We found that the expression of CDK4 and cyclin D1 were decreased when Bel-7402 and HepG2 cells were treated with Plac1-siRNA. Considering the reported role of Plac1-siRNA in inducing G1/S arrest and repressing CDK4 and cyclin D1 expression of breast cancer cells (10), it is conceivable that Plac1 regulates cell cycle distribution by controlling the levels of cell cycle-related proteins.

It is well known that cancer development and progression are attributed to destruction of the balance between cell proliferation and apoptosis (16). Since p53 possesses antitumorigenic function by regulating cell cycle and apoptosis $(17,18)$, we were interested if this key cell apoptosis-signaling protein was involved in the effect of Placl on apoptosis in HCC. Our findings in HepG2 and Bel-7402 cells showed that downregulation of Plac1 enhanced p53 expression, raises the possibility that Placl exerts biological behavior in HCC through suppressing the p53 level. Previous studies have demonstrated that the p53 protein can interact with the proteins of Bcl-2 family, which are classified as pro-apoptotic (Bax, Bad, Bid and Bim) and anti-apoptotic (Bcl-2 and Bcl-XL) (21) and induce apoptosis $(19,20)$. It has been shown that p53 induced apoptosis through repressing the transcription of Bcl-2 (22). Bel-7402 cells treated with Plac1 siRNA showed decreased expression of Bcl-2, which was inversely related to p53 expression, implying that downregulation of Bcl-2 expression in Bel-7402 cells might be suppressed by p53. This hypothesis is supported by previous studies showing that $\mathrm{Bcl}-2$ is negatively regulated by the tumor suppressor p53 $(23,24)$. Surprisingly, instead of upregulation, Bax expression was inhibited in Bel-7402 cells transfected with Plac1 siRNA. In fact, the $\mathrm{Bax} / \mathrm{Bcl}-2$ ratio is more important in determining the sensitivity to apoptotic stimuli in cancer cells than the expression of each protein individually (25). In contrast to Bel-7402 cells, we detected that silence of Plac1 strongly increased Bcl-2 expression in HepG2 cells, while Bax expression had a reduction. These data disclose that the mechanism of Plac1 on cell apoptosis in HepG2 and Bel-7402 cells are unlikely identical. Bax and Bcl-2 does not account for the Plac1-siRNA-mediated apoptosis in HepG2 cells, and another novel mechanism mediating apoptosis in HepG2 cells must be presented.

The correlation between Plac1 expression and metastasis of HCC in clinical specimens prompted our investigation into the role of Placl in cell motility and invasion. Concordantly, our data demonstrated that Plac1 promote migration and invasion of HCC, supported by the fact that knockdown of Plac1 suppressed Bel-7402 and HepG2 cell migration and invasion ability in vitro by using Transwell assay. Our further focus in the present study was to determine whether the changes in migration and invasion were caused by EMT. EMT is a pivotal physiological process contributing to cancer cell invasion and migration, including HCC $(26,27)$. It is by a dedifferentiation program that epithelial cells lose their epithelial signatures and gain mesenchymal characteristics (28). Downregulation of E-cadherin, an epithelial cell adhesion molecule, is regarded as the critical step of EMT $(29,30)$. Upregulation of mesenchymal 
marker vimentin and the most prominent suppressors of E-cadherin such as Snail, Twist, ZEB-1 and ZEB-2, which bind to E-boxes of E-cadherin promoter and suppress its transcription, are intensively involved in the process of EMT (29-32). In this study, downregulation of Placl elevated E-cadherin expression, accompanied by a distinct decreased of vimentin. This observation was reinforced by results with downregulation of Snail and Twist expression, which repress the expression of E-cadherin. Based on these observation, we conclude that Plac1 likely exerts its carcinogenic effect in HCC through development of EMT, thus, promoting cancer metastasis. We hope to gain more detailed insights into the molecular mechanism, in which Plac1 regulates EMT in our future research.

As PI3K/AKT pathway is an important regulatory signal for cancer process $(33,34)$, we wondered whether PI3K/Akt participated in Plac1-mediated biological behavior in HCC. We observed that phosphorylation of AKT was attenuated in Plac1-siRNA HCC cells, suggesting Placl might be involved in HCC carcinogenesis by activating PI3K/AKT signaling pathway. The finding is consistent with the result that phosphorylation of AKT was markedly decreased in breast cancer MCF-7 cells treated with Plac1 siRNA, and less prominent in another breast cancer cell line the BT-549 transfected with Plac1 siRNA, which lack PTEN. There is evidence to show that PTEN, which can bind to p53 and enhance p53-mediated functions, is a negative regulator of AKT $(34,35)$. Notably, the present study showed a positive effect of Plac1 on the AKT signaling pathway and a negative effect of Plac1 on p53. Little is known about Placl effect on PTEN level, but we hypothesize that Placl caused an activation of AKT pathway possibly through inhibition of PTEN and p53, thereby promoting HCC cell proliferation and metastasis. However, the detailed mechanism remains to be elucidated.

In conclusion, this study highlights that Placl is a critical factor for HCC development by facilitating HCC proliferation, cell cycle progression, cell migration through enhancement of EMT and resisting tumor cell apoptosis. Thus, Plac1 may serve as a promising target molecule for the development of novel therapeutic drugs in HCC.

\section{Acknowledgements}

The present study was supported by the Science and Technology Planning Project of Guangdong Province, China (no. 2012B031800427), and the Science and Technology Planning Project of Zhanjiang, Guangdong, China (no. 2013A01012).

\section{References}

1. Torre LA, Bray F, Siegel RL, Ferlay J, Lortet-Tieulent J and Jemal A: Global cancer statistics, 2012. CA Cancer J Clin 65: 87-108, 2015.

2. Maluccio M and Covey A: Recent progress in understanding, diagnosing, and treating hepatocellular carcinoma. CA Cancer J Clin 62: 394-399, 2012.

3. Cocchia M, Huber R, Pantano S, Chen EY, Ma P, Forabosco A, Ko MS and Schlessinger D: PLAC1, an Xq26 gene with placentaspecific expression. Genomics 68: 305-312, 2000.

4. Fant M, Weisoly DL, Cocchia M, Huber R, Khan S, Lunt T and Schlessinger D: PLAC1, a trophoblast-specific gene, is expressed throughout pregnancy in the human placenta and modulated by keratinocyte growth factor. Mol Reprod Dev 63: 430-436, 2002.
5. Fant M, Barerra-Saldana H, Dubinsky W, Poindexter B and Bick R: The PLAC1 protein localizes to membranous compartments in the apical region of the syncytiotrophoblast. Mol Reprod Dev 74: 922-929, 2007.

6. Chang WL, Yang Q, Zhang H, Lin HY, Zhou Z, Lu X, Zhu C, Xue LQ and Wang $\mathrm{H}$ : Role of placenta-specific protein 1 in trophoblast invasion and migration. Reproduction 148: 343-352, 2014.

7. Liu FF, Dong XY, Pang XW, Xing Q, Wang HC, Zhang HG, Li Y, Yin YH, Fant M, Ye YJ, et al: The specific immune response to tumor antigen CP1 and its correlation with improved survival in colon cancer patients. Gastroenterology 134: 998-1006, 2008

8. Dong XY, Peng JR, Ye YJ, Chen HS, Zhang LJ, Pang XW, Li Y, Zhang Y, Wang S, Fant ME, et al: Placl is a tumor-specific antigen capable of eliciting spontaneous antibody responses in human cancer patients. Int J Cancer 122: 2038-2043, 2008.

9. Silva WA Jr, Gnjatic S, Ritter E, Chua R, Cohen T, Hsu M, Jungbluth AA, Altorki NK, Chen YT, Old LJ, et al: PLAC1, a trophoblast-specific cell surface protein, is expressed in a range of human tumors and elicits spontaneous antibody responses. Cancer Immun 7: 18-25, 2007.

10. Koslowski M, Sahin U, Mitnacht-Kraus R, Seitz G, Huber C and Türeci O: A placenta-specific gene ectopically activated in many human cancers is essentially involved in malignant cell processes. Cancer Res 67: 9528-9534, 2007.

11. Liu F, Shen D, Kang X, Zhang C and Song Q: New tumour antigen PLAC1/CP1, a potentially useful prognostic marker and immunotherapy target for gastric adenocarcinoma. J Clin Pathol 68: 913-916, 2015.

12. Ghods R, Ghahremani MH, Madjd Z, Asgari M, Abolhasani M, Tavasoli S, Mahmoudi AR, Darzi M, Pasalar P, Jeddi-Tehrani M, et al: High placenta-specific 1/low prostate-specific antigen expression pattern in high-grade prostate adenocarcinoma. Cancer Immunol Immunother 63: 1319-1327, 2014.

13. Satyanarayana A and Kaldis P: Mammalian cell-cycle regulation: Several Cdks, numerous cyclins and diverse compensatory mechanisms. Oncogene 28: 2925-2939, 2009.

14. Hochegger H, Takeda S and Hunt T: Cyclin-dependent kinases and cell-cycle transitions: Does one fit all? Nat Rev Mol Cell Biol 9: 910-916, 2008.

15. Fung TK and Poon RY: A roller coaster ride with the mitotic cyclins. Semin Cell Dev Biol 16: 335-342, 2005.

16. Evan GI and Vousden KH: Proliferation, cell cycle and apoptosis in cancer. Nature 411: 342-348, 2001.

17. Avery-Kiejda KA, Bowden NA, Croft AJ, Scurr LL, Kairupan CF, Ashton KA, Talseth-Palmer BA, Rizos H, Zhang XD, Scott RJ, et al: P53 in human melanoma fails to regulate target genes associated with apoptosis and the cell cycle and may contribute to proliferation. BMC Cancer 11: 203-219, 2011.

18. Zhang YQ, Xiao CX, Lin BY, Shi Y, Liu YP, Liu JJ, Guleng B and Ren JL: Silencing of Pokemon enhances caspase-dependent apoptosis via fas- and mitochondria-mediated pathways in hepatocellular carcinoma cells. PLoS One 8: e68981, 2013.

19. Zand H, Rhimipour A, Bakhshayesh M, Shafiee M, Nour Mohammadi I and Salimi S: Involvement of PPAR-gamma and p53 in DHA-induced apoptosis in Reh cells. Mol Cell Biochem 304: 71-77, 2007.

20. Sankari SL, Masthan KMK, Babu NA, Bhattacharjee T and Elumalai M: Apoptosis in cancer - an update. Asian Pac J Cancer Prev 13: 4873-4878, 2012.

21. Reed JC: Bcl-2 family proteins: Regulators of apoptosis and chemoresistance in hematologic malignancies. Semin Hematol 34 (Suppl 5): 9-19, 1997.

22. Yee KS and Vousden KH: Complicating the complexity of p53. Carcinogenesis 26: 1317-1322, 2005.

23. Nakazawa K, Dashzeveg N and Yoshida K: Tumor suppressor p53 induces miR-1915 processing to inhibit Bcl-2 in the apoptotic response to DNA damage. FEBS J 281: 2937-2944, 2014.

24. Borghetti G, Yamaguchi AA, Aikawa J, Yamazaki RK, de Brito GA and Fernandes LC: Fish oil administration mediates apoptosis of Walker 256 tumor cells by modulation of p53, Bcl-2, caspase-7 and caspase-3 protein expression. Lipids Health Dis 14: 94-98, 2015.

25. Raisova M, Hossini AM, Eberle J, Riebeling C, Wieder T, Sturm I, Daniel PT, Orfanos CE and Geilen CC: The Bax/Bcl-2 ratio determines the susceptibility of human melanoma cells to CD95/Fas-mediated apoptosis. J Invest Dermatol 117: 333-340, 2001.

26. Maheswaran T and Rushbrook SM: Epithelial-mesenchymal transition and the liver: Role in hepatocellular carcinoma and liver fibrosis. J Gastroenterol Hepatol 27: 418-420, 2012. 
27. Li YM, Xu SC, Li J, Han KQ, Pi HF, Zheng L, Zuo GH Huang XB, Li HY, Zhao HZ, et al: Epithelial-mesenchymal transition markers expressed in circulating tumor cells in hepatocellular carcinoma patients with different stages of disease. Cell Death Dis 4: e831, 2013.

28. Panebianco C, Saracino C and Pazienza V: Epithelialmesenchymal transition: Molecular pathways of hepatitis viruses-induced hepatocellular carcinoma progression. Tumour Biol 35: 7307-7315, 2014

29. Zheng H and Kang Y: Multilayer control of the EMT master regulators. Oncogene 33: 1755-1763, 2014.

30. Zhai X, Zhu H, Wang W, Zhang S, Zhang Y and Mao G: Abnormal expression of EMT-related proteins, S100A4, vimentin and E-cadherin, is correlated with clinicopathological features and prognosis in HCC. Med Oncol 31: 970, 2014.
31. Peinado H, Olmeda D and Cano A: Snail, Zeb and bHLH factors in tumour progression: An alliance against the epithelial phenotype? Nat Rev Cancer 7: 415-428, 2007.

32. Peinado H, Portillo F and Cano A: Transcriptional regulation of cadherins during development and carcinogenesis. Int J Dev Biol 48: 365-375, 2004.

33. Faes S and Dormond O: PI3K and AKT: Unfaithful partners in cancer. Int J Mol Sci 16: 21138-21152, 2015.

34. Martini M, De Santis MC, Braccini L, Gulluni F and Hirsch E: PI3K/AKT signaling pathway and cancer: An updated review. Ann Med 46: 372-383, 2014.

35. Trotman LC, Wang X, Alimonti A, Chen Z, Teruya-Feldstein J, Yang H, Pavletich NP, Carver BS, Cordon-Cardo C, ErdjumentBromage $\mathrm{H}$, et al: Ubiquitination regulates PTEN nuclear import and tumor suppression. Cell 128: 141-156, 2007. 